\title{
Adaptive Carrier-Sensing for Throughput Improvement in IEEE 802.11 Networks
}

\author{
Ehsan Haghani, Michael N. Krishnan, and Avideh Zakhor \\ Video and Image Processing Lab \\ Department of Electrical Engineering and Computer Science \\ University of California, Berkeley \\ Email: \{Haghani, Mkrishna, Avz $\}$ eecs.berkeley.edu
}

\begin{abstract}
As a Carrier Sense Multiple Access (CSMA) network, the performance of IEEE 802.11 networks highly depends on the accuracy of the carrier sensing procedure. However, conventional carrier sensing approaches suffer from the well known hidden and exposed node problems, adversely affecting aggregate throughput of the IEEE 802.11 networks. In this paper, we propose a novel scheme through which each station can adaptively select its Carrier Sense Threshold (CST) in order to mitigate the hidden/exposed node problems. The basic idea behind our approach is for the Access Point (AP) to periodically transmit a Busy/Idle (BI) signal to all the stations. Individual stations then use the BI signal from the AP together with their own local BI signal in order to adjust their CST. We use NS-2 simulations to show that our approach can enhance the aggregate throughput by as much as $50 \%$.
\end{abstract}

\section{INTRODUCTION}

Wireless Local Area Networks (WLANs) are pervasively implemented to provide users with broadband wireless connectivity. The need for efficient methods to share the limited resources in WLANs has called for extensive research on resource sharing and spatial reuse. In IEEE 802.11 networks, Distributed Coordination Function (DCF) or CSMA/CA deploys carrier sensing to determine the availability of the shared medium prior to transmitting. Two different approaches to carrier sensing are supported by DCF: Virtual Carrier Sensing (VCS) and Physical Carrier Sensing (PCS). In VCS, each node uses the request to send/clear to send (RTS/CTS) messages to reserve the access to channel prior to transmission. It is assumed that nodes surrounding the destination receive the CTS message, thus avoiding collision. However, this approach is rarely used in practice because of its large overhead. Moreover, there are several scenarios where RTS/CTS results in lower throughput [1]. In PCS, each node examines the status of the channel prior to transmission by comparing the measured received energy in the wireless channel with the Carrier Sense Threshold (CST). The node attempts channel access only if the measured energy level of the channel is less than the CST indicating that the channel is idle; otherwise, the node backs off and waits for a random period of time.

Even though PCS does not impose any overhead on the network, it is not an accurate indicator of the channel status as sensed by the receiver. This inherent flaw of PCS causes the well known hidden and exposed node problems. The hidden node problem occurs when the receiver cannot successfully receive the transmitted packet due to interference caused by another transmission initiated by a node located outside the sensing range of the transmitter. The transmitter cannot sense the hidden node if its CST value is larger than the received energy level of the signal transmitted by the hidden node. The hidden node problem causes collisions at the receiver, thus reducing the throughput of the network. Conversely, the exposed node problem occurs when a transmitter needlessly avoids transmission in order to avoid interference with another concurrent transmission, even though the interference at its receiver is lower than the decoding threshold. In this case, CST of the transmitter is small enough that it senses faraway transmissions, thus unnecessarily deferring transmission. In general, the exposed node problem reduces the throughput of the network by needlessly deferring transmissions that could have successfully been received. The value of CST at each station has a direct impact on its perceived status of the channel locally; as such, there is an inherent tradeoff between the number of hidden and exposed nodes depending on the CST value chosen by a given station. While decreasing CST makes the station sense nodes further away and reduces the number of hidden nodes, it also increases the number of exposed nodes unnecessarily sensed by the station. Therefore, for each node there is an optimum CST that balances the impact of the hidden and exposed nodes.

The impact of CST on the performance of the network has been studied by a number of researchers in recent years. Ma et al. [2] propose a centralized algorithm for adjusting the CST based on loss differentiation. In their algorithm a central controller, such as an Access Point (AP), periodically receives feedback from all stations and calculates the new CST to be used by all the nodes in the network. In this work, since all the nodes use the same CST, the network is unlikely to reach the maximum achievable throughput. Nadeem and Ji [3] propose an enhanced DCF protocol that incorporates the location information to increase the spatial reuse. In their work, it is assumed that each node knows its own location and includes this information in each transmission so that all overhearing stations can predict whether their transmissions affect the ongoing transmission. Zhu et al. [4] propose an algorithm to adjust the CST so as to maximize the aggregate 
throughput given a minimum required Signal to Noise Ratio (SNR). Their proposed adaptive algorithm uses the packet error rate to update the CST. Park et al. [5] propose an adaptive algorithm for updating CST. This work also uses packet error rate to update CST, but similar to [4], it does not differentiate between various causes of packet loss i.e., collisions and channel fadings; this is problematic because CST only affects collision, and not fading. Krishnan et al. [6] have illustrated the importance of differentiating between various loss types, and have proposed a method in which the stations estimate the probabilities of various types of loss. The basic idea behind [6] is for the AP to periodically transmit a busy/idle (BI) signal indicating the status of the channel at the AP to the stations. The stations use that signal together with their own local BI signal in order to estimate staggered and direct collision probabilities as well as fading loss probability.

Inspired by [6], in this paper, we propose a method through which stations can determine the channel status at their corresponding receivers. In our approach, similar to [6], we assume the AP to periodically broadcast its BI signal to the stations. Each station uses this signal together with its own local BI signal to optimally determine its CST. Therefore, spatial information dissemination by the AP results in a distributed, adaptive, CST algorithm that strikes a balance between sensing hidden and exposed nodes in order to maximize the throughput.

The outline of the remainder of the paper is as follows. Section II describes our proposed mechanism for information dissemination in the network. In Section III, we explain our proposed adaptive algorithm for updating CST. We present NS-2 simulation results in Section IV. Concluding remarks are included in Section V.

\section{Spatial Information DisseminAtion}

In our proposed approach, all the stations and access points record their received energy level at all time slots of $10 \mu \mathrm{sec}$ duration. The APs compare the received energy at each time slot with their current CSTs. If the received energy is higher than the CST, the AP assumes that time slot to be busy; otherwise, that time slot is assumed to be idle. Thus, each AP generates a binary signal representing its experienced channel occupancy in the terms of a busy/idle signal, and periodically broadcasts this to its associated stations every few seconds. In the BI signal, the AP includes the channel occupancy information for all times since the transmission of the last broadcast BI signal. As shown in [6], the transmission overhead of the BI signal is only about $3 \%$ of the aggregate throughput of $802.11 \mathrm{~b}$ network. Assuming each node records its local energy level at each time slot in a double precision variable of 8 bytes, the amount of needed memory at each node for 3 seconds of recording is about $2.4 M B$.

As explained earlier, stations can not accurately predict the status of the channel at the AP in the PCS approach, due to the hidden and exposed nodes. Rather, stations must decide whether or not to transmit based on observations of their local channel. In particular, when the received energy of their channel is less than their CST, stations transmit to the AP, otherwise they back off. However, successful reception at the AP depends on the AP's channel status in that time slot. The intuitive idea behind generating and broadcasting the BI signal from the AP to the stations is to provide them with information about the channel status at the AP. Therefore, it is desirable for each station to choose its CST such that its resulting local BI signal mimics the BI signal of the receiving AP. In Section III, we propose a method for the stations to adaptively select the CST so as to achieve this.

\section{AdAptive CARrier Sensing Algorithm}

Each station can deduce its channel occupancy at a time slot by comparing its received energy level at that time slot with its carrier sense threshold. If the energy level is higher than the threshold, the station assumes the channel to be busy in that time slot; otherwise, the channel is assumed to be idle. Thus, the value of the CST at each station directly affects the existence and number of the hidden and exposed nodes experienced by it. As explained earlier, due to the trade-off between the hidden and exposed nodes, increasing the carrier sense threshold at the station decreases the number of the exposed nodes while increasing the number of hidden nodes. On the other hand, choosing a lower carrier sense threshold decreases the number of the hidden nodes while it increases the number of the exposed nodes.

Stations can compare the received BI signal from the AP with their own energy profile over the sampling period to gain knowledge about the existence of hidden and exposed nodes. For example, if for a given time slot the station senses the channel to be busy but the AP claims otherwise, the station can deduce that the activity of an exposed node in that time slot has resulted in this difference. On the other hand, the station can detect the existence of a hidden node if it senses the channel idle at a time slot while the AP reports a busy channel. We use this feature of the busy/idle signal to derive an adaptive algorithm for choosing the carrier sense threshold.

Fig. 1 shows a sample schematic of the recorded energy profile of a station and the binary BI signal transmitted by the AP. The energy profile at the station has multiple levels while the BI signal of the AP is a binary signal. The highlighted slots in the station energy profile indicate transmissions of exposed nodes and the highlighted slots in the BI signal of AP indicate transmissions of hidden nodes. $n_{01}$ and $n_{10}$ represent the number of slots affected by transmissions of hidden and exposed nodes respectively. Increasing the CST from $L_{1}$ to $L_{4}$ in Fig. 1(a) to Fig. 1(d) changes the total number of slots affected by transmissions from the hidden and exposed nodes, $n_{01}+n_{10}$, from 4 to 3 , to 5 , and to 8 with the minimum occurring at $L_{1}<C S T<L_{2}$. Since both hidden and exposed nodes can potentially lower the throughput of the network, it is desirable for each station to choose its CST so as to minimize the number of time slots in which its hidden and exposed nodes affect its transmissions [7]. Since different stations experience different number of hidden or exposed nodes according to their 


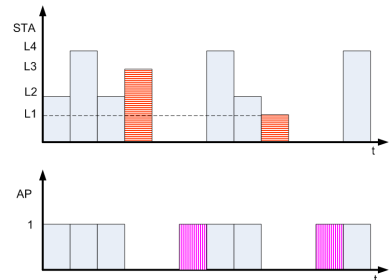

(a)
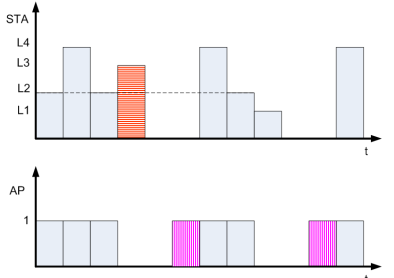

(b)
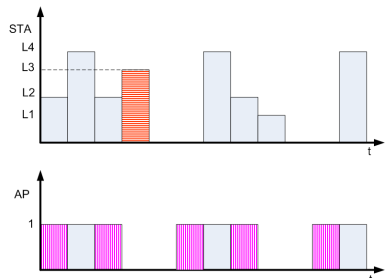

(c)

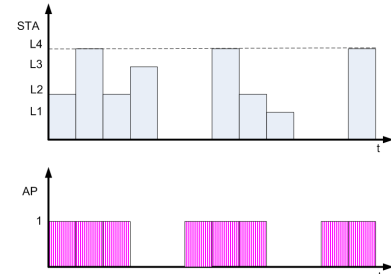

(d)

Fig. 1. BI signal of AP shown at the bottom and energy profile of a station shown on top. (a) $C S T<L_{1} ; n_{01}=2 ; n_{10}=2$. (b) $L_{1}<C S T<L_{2}$; $n_{01}=2 ; n_{10}=1$. (c) $L_{2}<C S T<L_{3} ; n_{01}=5 ; n_{10}=1$. (d) $C S T>L_{4} ; n_{01}=8 ; n_{10}=0$.

locations, using a constant CST for all nodes is not optimal. We elaborate more on this later in Section IV.

In a network with multiple access points, we assume each station connects to the AP with the highest received signal power. Each access point broadcasts its binary BI signal every $\Delta$ seconds to indicate its channel occupancy for all time slots of $\delta$ second duration. Each station records the received energy level for all time slots in the period of $\Delta$ seconds as well. By comparing the energy level with the carrier sense threshold $\gamma$, the station generates its own BI signal $B I_{\text {station. }}^{\gamma}$. Specifically, for $p, q \in\{0,1\}$, we define

$$
P_{p q}^{\gamma}(t)=\operatorname{Pr}\left\{B I_{\text {station }}^{\gamma}(t)=p, B I_{A P}(t)=q\right\}
$$

where $t$ is the time slot number, and $1 \leq t \leq\left\lfloor\frac{\Delta}{\delta}\right\rfloor$. It is assumed that $p$ or $q$ are equal to 0 if the channel is idle, and 1 if the channel is busy. As mentioned earlier, the activities of hidden or exposed nodes result in a difference in the observed channel occupancy at the AP and station. In particular the numbers of transmissions of hidden and exposed nodes are proportional to $P_{01}^{\gamma_{i}}$ and $P_{10}^{\gamma_{i}}$ respectively for $C S T=\gamma_{i}$. Specifically,

$$
\begin{aligned}
& \mid \text { Transmissions of Hidden Nodes } \mid \propto P_{01}^{\gamma_{i}} \\
& \mid \text { Transmissions of Exposed Nodes } \mid \propto P_{10}^{\gamma_{i}}
\end{aligned}
$$

To maximize the throughput of the network, it is reasonable to minimize the number of transmissions of hidden and exposed nodes for each station by changing its CST. We therefore define the optimization function $F$ as the sum of the number of hidden nodes and exposed nodes for each station during $\Delta$ :

$$
\gamma_{o p t}=\arg \min _{\gamma_{i}} F_{i}\left(\gamma_{i}\right)
$$

where

$$
F_{i}\left(\gamma_{i}\right)=P_{01}^{\gamma_{i}}+P_{10}^{\gamma_{i}}
$$

Since the AP broadcasts its BI signal every $\Delta$ seconds, each station can incorporate an adaptive algorithm for adjusting its CST every $\Delta$ seconds i.e. every iteration. In our proposed adaptive algorithm, each station listens to the channel and records the energy level at all time slots. After receiving the binary busy/idle signal from the AP, the station performs an exhaustive search to choose the best carrier sense threshold, $\gamma_{\text {opt }}$ from a finite set of possible values for the carrier sense,
$\Gamma=\left\{\gamma_{1}, \gamma_{2}, \ldots, \gamma_{N}\right\}$. For each value of $\gamma_{i}$, the station has to compute the corresponding $P_{01}^{\gamma_{i}}$ and $P_{10}^{\gamma_{i}}$ to evaluate the optimization function $F_{i}\left(\gamma_{i}\right)$.

As shown in Section IV, it is possible that different carrier sense thresholds result in similar values of the optimization function. To avoid excessive variations of CST, we allow the station to adhere to its current CST value if the percentage change between the new optimum value of $F$ and the function value corresponding to the current CST is less than a constant value, $\rho$. Furthermore, if the percentage change is higher than $\rho$, the station chooses the closest CST value to its current CST whose difference from the optimum CST is less than $\rho$ percent. Since variation of CST for each node affects the performance of other nodes in the network, this approach makes the network evolve in a more stable fashion, and prevents chaotic situations in which all the nodes significantly change their CST in every iteration. The pseudo-code for the adaptive selection of the carrier sense threshold is shown in Algorithm 1.

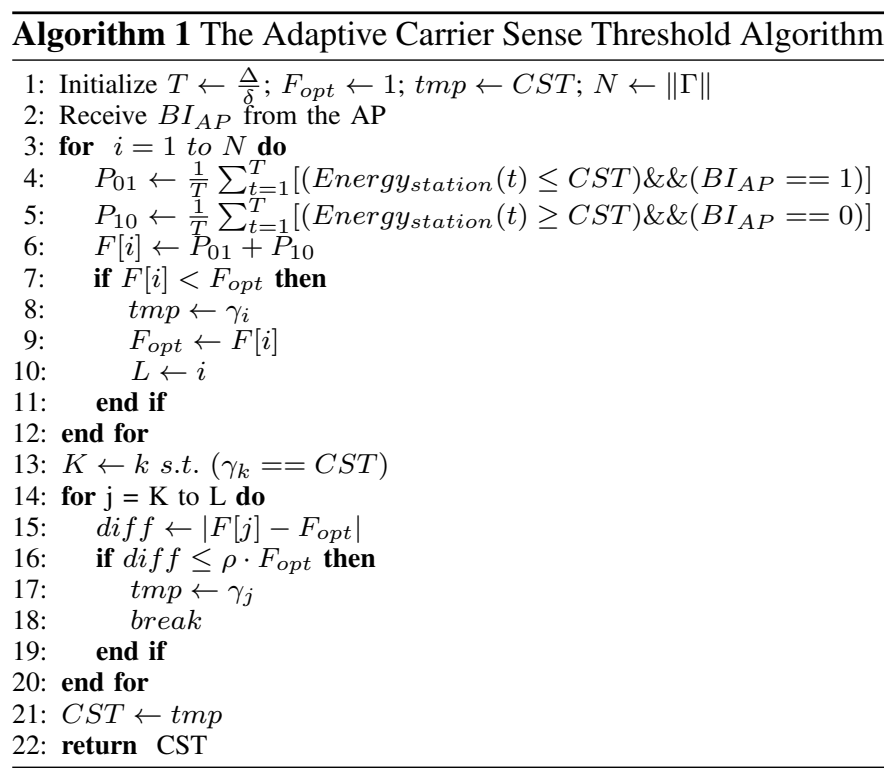

\section{Simulation Results}

In this section, we evaluate the performance of our proposed solution for selecting CST. We study an IEEE 802.11b network in which all the stations implement the adaptive carrier sense 
TABLE I

Simulation PARAMETERS

\begin{tabular}{|l|l|}
\hline Parameter & Value \\
\hline \hline No. Stations & 50 \\
\hline No. AP & 7 \\
\hline Area & $500 \times 450(\mathrm{~m})$ \\
\hline Carrier Freq. & $2.4 \mathrm{GHz}$ \\
\hline Transmit Power & $32 \mathrm{~mW}$ \\
\hline Path Loss Factor & 4 \\
\hline Bitrate & $11 \mathrm{Mbps}$ \\
\hline$\Delta$ & $3 \mathrm{sec}$ \\
\hline$\delta$ & $10 \mu \mathrm{sec}$ \\
\hline$\rho$ & $5 \%$ \\
\hline
\end{tabular}

TABLE II

CST AND COVERAGE

\begin{tabular}{|l|l|l|l|l|l|}
\hline CST (W) & $2 \mathrm{e}-13$ & $7 \mathrm{e}-14$ & $2 \mathrm{e}-14$ & $7 \mathrm{e}-15$ & $2 \mathrm{e}-16$ \\
\hline Radius (M) & 63 & 82 & 112 & 272 & 354 \\
\hline
\end{tabular}

threshold algorithm, and compare its aggregate throughput to that of the same network with all stations using a constant CST. The APs use a constant CST in both cases. We modify the NS2 simulation package to (a) allow stations record the energy level of the receiving channel at all time slots, and (b) allow the APs compute their BI signals and to periodically broadcast them to their associated stations. The network and simulation parameters are shown in Table I. The position of the 7 APs is assumed to be unchanged from one simulation scenario to the next, while the random locations of the stations vary from one scenario to the next. The distance between adjacent APs is about 172 meters.

Since the APs locations are fixed, we only study the CS ranges which cover most of the area without APs overhearing each other. Table II shows the simulated CSTs in watts and their corresponding coverage radius in meters.

A sample schematic of nodes and access points for a given simulation scenario is depicted in Fig. 2. The centers of the depicted circles represent the locations of the stations; the diameter of each circle is proportional to the percentage of throughput difference in each node when adaptive and fixed CST selection schemes are implemented. The empty blue circles show enhancement in throughput while red colored circles show loss of the throughput for that node.

Fig. 3 presents the histogram of percentage change in the throughput of each node for adaptive CST algorithm as compared to constant $C S T=7 \times 10^{-14}$. The histogram data is collected from 10 rounds of 60 second simulations, each of which includes 50 stations, i.e. overall 500 samples. In each simulation scenario, we use a different set of random locations for the stations, but the positions of APs are fixed. The figure

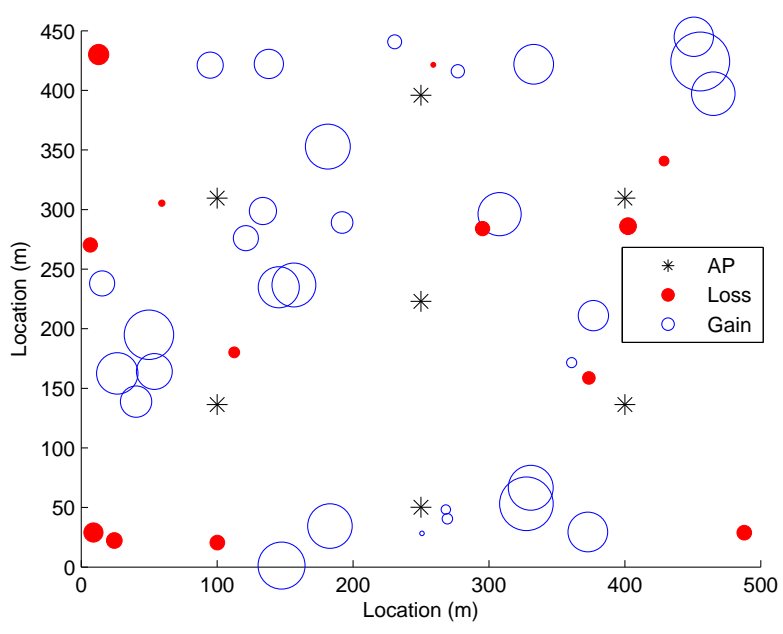

Fig. 2. Throughput variation vs. location with adaptive CST.

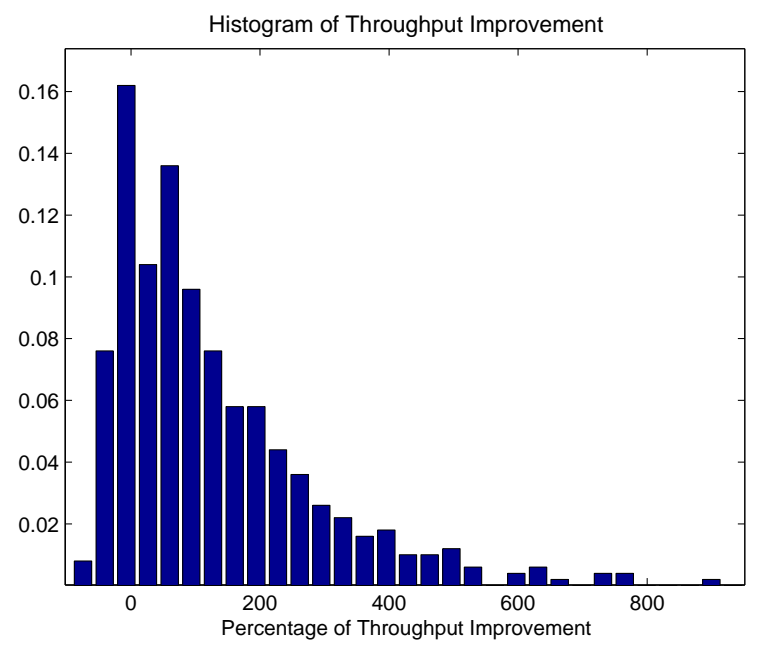

Fig. 3. Histogram of percentage change in the throughput of stations for 10 scenarios with 50 stations per scenario.

shows that almost $90 \%$ of the nodes have improved their throughput. The median and average of throughput improvement of all stations are about $81 \%$ and $131 \%$ respectively. Our simulations have shown that those stations losing throughput could have improved their throughput if they were the only station in networking applying the adaptive CST algorithm and all other stations had used a constant CST. Since stations do not have any a priori information about activities of other stations, there is high motivation for the stations to incorporate the adaptive solution in the hope of improving their throughput.

In our next experiment, we examine the aggregate throughput improvement for the various AP CST values listed in Table II. In adaptive approach, all stations use the adaptive CST algorithm but the APs use constant CST and in nonadaptive approach, all stations and APs use the same constant CST. Fig. 4 compares the aggregate throughput of the network 


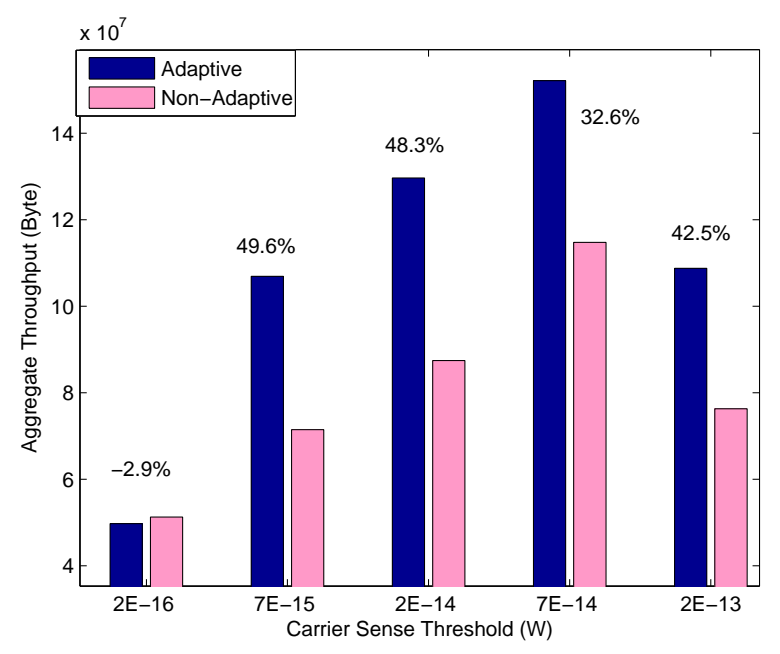

Fig. 4. Aggregate throughput comparison for different CST values.

for both adaptive and non-adaptive schemes as a function of the default CST. Each value is obtained by averaging at least 10 different simulation scenarios of 60 seconds with random station locations varying from one scenario to the next. The percentage of throughput enhancement for each case is also presented in Fig. 4. As seen, the adaptive CST selection algorithm can increase the aggregate throughput of the network up to $50 \%$ for $C S T=7 \times 10^{-14}$; or decrease it by about $2 \%$ when $C S T=2 \times 10^{-16}$; in the latter case, each AP can sense the transmissions of at least 5 other APs and most of their surrounding stations. As such, it is not useful to study CST adaptation for stations in this case, because the APs have very poor spatial reuse.

To investigate the fairness of the adaptive CST algorithm, we study the log-throughput factor [8] in the network.

$$
\Phi=\frac{1}{|S|} \sum_{j=1}^{|S|} \log \left(\phi_{j}\right)
$$

where $\Phi$ is the log-throughput factor of the network, $\phi_{j}$ is the throughput of station $j$, and $|S|$ is the number of stations in the network. By studying the log-throughput factor, it is possible to determine whether the throughput enhancement of the network is due to unfairly increasing the throughput of the stations with better quality physical channels, or conversely resulting from fairly increasing the throughput of all nodes. Fig. 5 presents the log-throughput factor for each default CST. As seen, the average of log-throughput is increased in all scenarios, indicating that the adaptive CST algorithm behaves fairly.

We study the probability of packet loss and number of transmission attempts for the stations in both adaptive and nonadaptive scenarios. Fig. 6 is a scatter plot of the probabilities of packet losses for all 500 stations in 10 simulation scenarios of 60 seconds each when the default CST is $7 \times 10^{-14}$. Since the majority of the points are located to the left of the 45 degree line, we conclude that adaptive CST algorithm

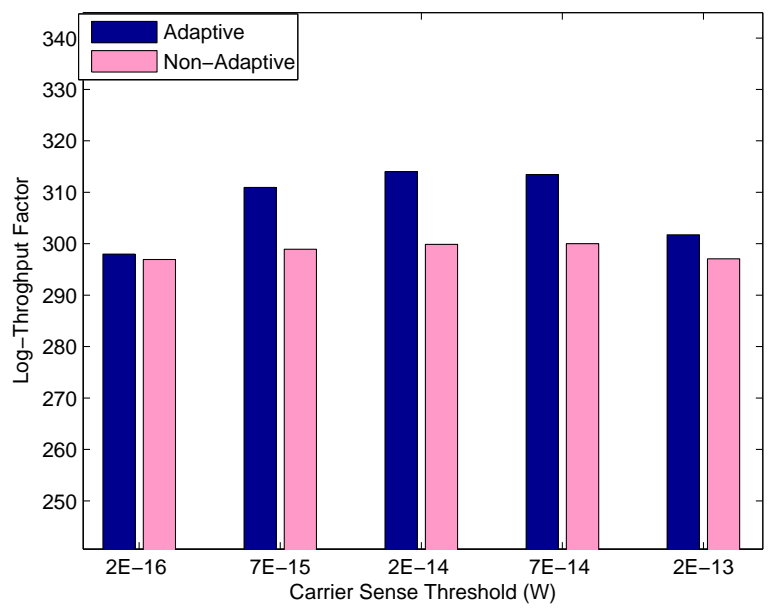

Fig. 5. Log-Throughput factor comparison.

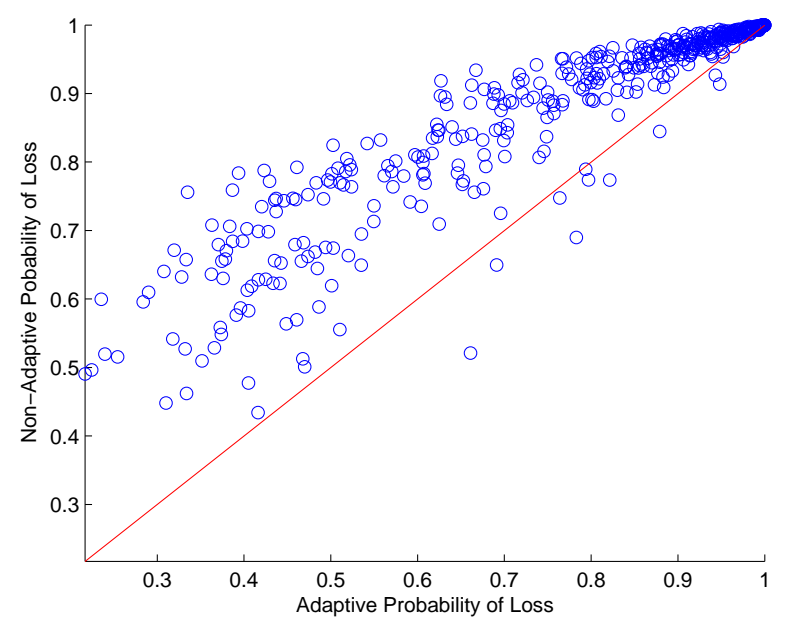

Fig. 6. Probability of packet loss for 500 stations from 10 simulation scenarios.

decreases the probability of packet loss. Since simulations for adaptive and non-adaptive scenarios are conducted under similar PHY conditions, decrease in the probability of packet loss primarily results from decrease in the collision probability. Fig. 7 compares the number of transmission attempts of stations throughout the simulation time for 10 scenarios with 50 stations per scenario. As seen, the number of transmission attempts is decreased for most stations using the adaptive CST algorithm. From Figs 6 and 7 we conclude that using adaptive CST algorithm improves the aggregate throughput by decreasing the probability of collision in the network.

As explained in Section III, we do not change the CST in the adaptive CST algorithm unless the percentage change in the optimization function, $F$, is larger than a constant threshold, $\rho$. This is because function $F$ exhibits shallow variations around the minimum value in many cases. Furthermore, changing the CST too frequently may prohibit the nodes from reaching their 


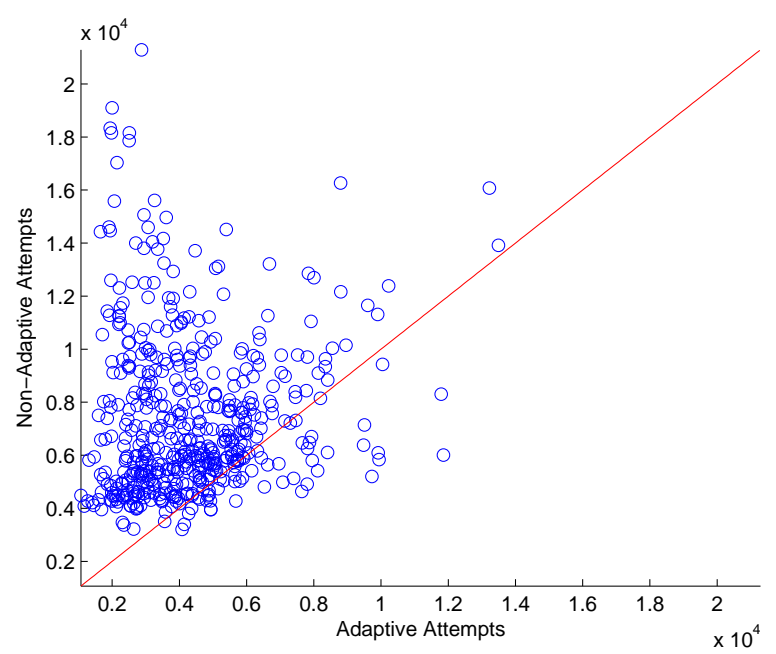

Fig. 7. Transmission attempts for 500 nodes from 10 simulation scenarios.

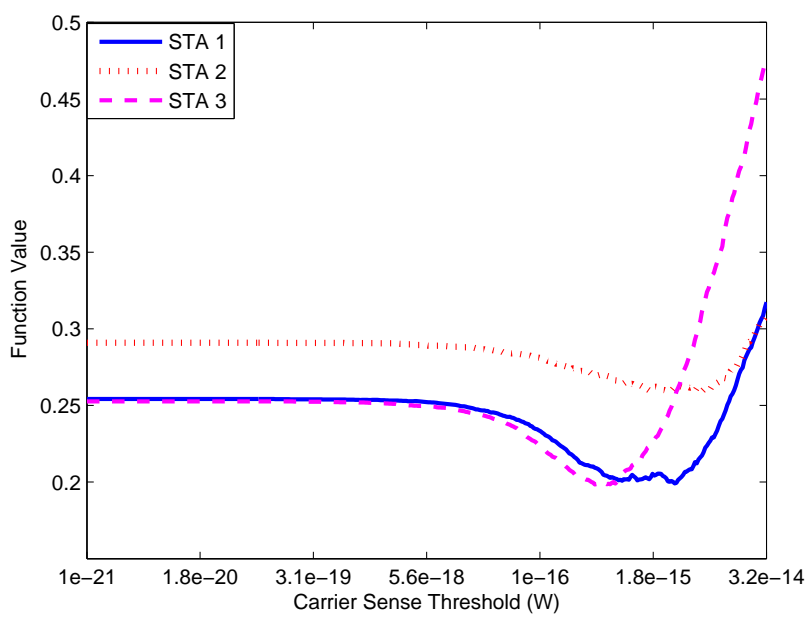

Fig. 8. $F$ as a function of CST.

optimum values since changing the CST in one node affects the performance of other nodes which may in turn trigger them to change their CST in response. Fig. 8 shows the values of the optimization function, $F$, for three different stations with respect to the possible CST values in one round of CST updating. As seen, the value of the function shows only small variations around the minimum value in some cases. Fig. 9 depicts the variation of the CST vs. simulation time for three different nodes in the network for the adaptive CST algorithm. As seen, the CST values vary smoothly and infrequently over time.

\section{CONCLUSION}

In this paper, we have proposed an adaptive and distributed algorithm for choosing the carrier sense threshold of the nodes in IEEE 802.11 networks. In our approach, nodes use the channel occupancy information periodically broadcast by the access point to adaptively choose the carrier sense threshold.

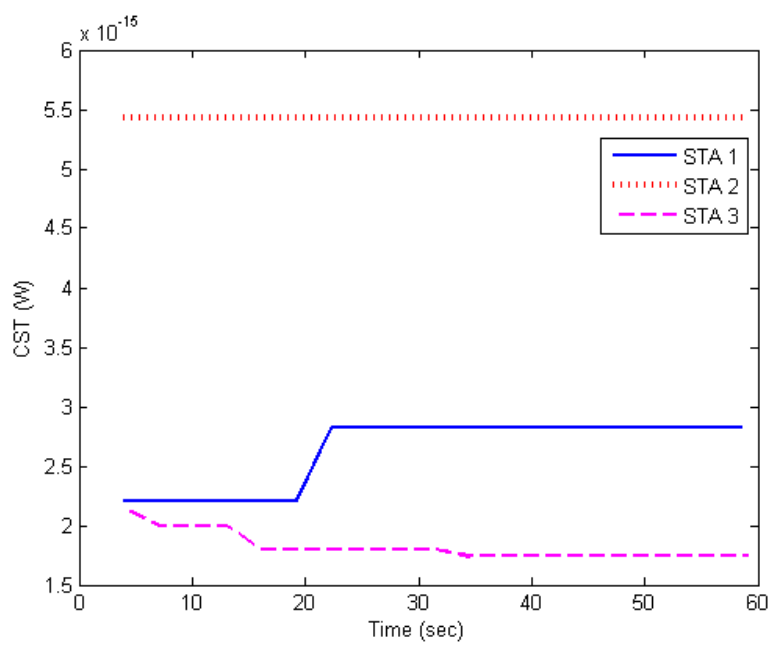

Fig. 9. Carrier sense variation as a function of time.

We have discussed the trade-off between the number of the hidden and exposed nodes and their relation to the carrier sense threshold. We have used NS-2 simulations to show that our approach can increase the aggregate throughput of the network by as much as $50 \%$.

\section{REFERENCES}

[1] G. Anastasi, E. Borgia, M. Conti, and E. Gregori, "WiFi in ad hoc mode: a measurement study," in Proceedings of the Second IEEE Annual Conference on Pervasive Computing and Communications, PerCom 2004, March 2004, pp. 145-154.

[2] H. Ma, R. Vijayakumar, S. Roy, and J. Zhu, "Optimizing 802.11 wireless mesh networks based on physical carrier sensing," IEEE/ACM Transactions on Networking, vol. 17, no. 5, pp. 1550-1563, Oct. 2009.

[3] T. Nadeem and L. Ji, "Location-aware IEEE 802.11 for spatial reuse enhancement," IEEE Transactions on Mobile Computing, vol. 6, no. 10, pp. 1171-1184, Oct. 2007.

[4] J. Zhu, X. Guo, L. Lily Yang, W. Steven Conner, S. Roy, and M. M. Hazra, "Adapting physical carrier sensing to maximize spatial reuse in 802.11 mesh networks," Wireless Communications and Mobile Computing Journal, vol. 4, no. 8, pp. 933-946, 2004.

[5] K.-J. Park, L. Kim, and J. Hou, "Adaptive physical carrier sense in topology-controlled wireless networks," IEEE Transactions on Mobile Computing, vol. 9, no. 1, pp. 87-97, Jan. 2010.

[6] M. N. Krishnan, S. Pollin, and A. Zakhor, "Local estimation of probabilities of direct and staggered collisions in 802.11 WLANs," in Proceedings of the IEEE GLOBECOM 2009, Dec. 2009.

[7] Z. Zeng, Y. Yang, and J. Hou, "How physical carrier sense affects system throughput in IEEE 802.11 wireless networks," in Proceedings of the IEEE INFOCOM 2008, April 2008, pp. 1445-1453.

[8] F. P. Kelly, A. K. Maulloo, and D. K. H. Tan, "Rate control for communication networks: Shadow prices, proportional fairness and stability," The Journal of the Operational Research Society, vol. 49, no. 3, pp. 237-252, Mar. 1998. 